Muñoz-Cantero, J.M. \& Pozo, C. (2014). El escenario de la calidad en la Universidad española: de dónde venimos y hacia dónde vamos. Revista Electrónica Interuniversitaria de Formación del Profesorado, 17 (3), 1-16.

DOI: http://dx.doi.org/10.6018/reifop.17.3. 204011

\title{
El escenario de la calidad en la Universidad española: de dónde venimos y hacia dónde vamos
}

\author{
Jesús Miguel Muñoz-Cantero ${ }^{(1)}$, Carmen Pozo Muñoz ${ }^{(2)}$ \\ (1) Universidad de A Coruña, (2) Universidad de Almería
}

\section{Resumen}

Durante la década de los 90 del pasado siglo, toman relevancia en la educación universitaria española conceptos y visiones hasta entonces desconocidos: sistema de indicadores, evaluación de programas/títulos, aseguramiento de la calidad, homologación, acreditación... La escasa permeabilidad del sistema no posibilitó la impregnación de la cultura de la calidad en todos sus estratos o niveles y en todas las Unidades. La Declaración de Bolonia, en junio de 1999, fue el impulso decisivo para la construcción del Espacio Europeo de Educación Superior (EEES) en España. Desde entonces se ha diseñado la moderna arquitectura normativa para mejorar su calidad docente investigadora y de gestión. La Ordenación de las Enseñanzas Universitarias Oficiales recoge la necesidad de establecer criterios que faciliten la evaluación, certificación y acreditación. El establecimiento de un Sistema de Garantía de Calidad (SGC) es de obligado cumplimiento para el proceso de Verificación y Acreditación de los Títulos. Iniciada la implantación de las enseñanzas correspondientes, los órganos de evaluación que la ley ha establecido, son los encargados de llevar a cabo el seguimiento partiendo del SGC adoptado y que orienta la futura acreditación de los títulos. Hoy por hoy, el protocolo de renovación de la acreditación de títulos está aún por dilucidar de manera definitiva.

\section{Palabras clave}

Espacio Europeo de Educación Superior; Calidad; verificación; acreditación. 


\title{
The scenario of quality in Spanish University: where we came from and where we are going
}

\begin{abstract}
During the 90 s of last century, become significant in the Spanish university education concepts and visions hitherto - unknown: System of indicators, program evaluation, qualifications, quality assurance, accreditation,... The low permeability of the system does not it enabled the impregnation quality culture in all its strata or levels and in all Units. The Bologna Declaration in June 1999, was decisive for the construction of the European Higher Education Area (EHEA) in Spain. Since then he has designed modern architecture rules to improve their teaching, research and management quality. The Organization of Official University Education recognizes the need to establish criteria to facilitate the evaluation, certification and accreditation. The establishment of a Quality Assurance System (QMS) is mandatory for the process of verification and accreditation of Titles. Initiated the implementation of the relevant teachings legalist establish evaluation committees, be held on the basis of this monitoring system to guide the accreditation of qualifications. While this is being done, regulation for re-accreditation of official title, today, is not defined.
\end{abstract}

\section{Key words}

European Higher Education Area; Quality; verification; accreditation.

\section{Introducción}

\section{Los orígenes en España}

Durante la década de los 90 del pasado siglo, toman relevancia en la educación universitaria conceptos y visiones hasta entonces desconocidos. Sistemas de indicadores, evaluación de programas/títulos, aseguramiento de la calidad, acreditación... Estos conceptos y visiones se hacen presentes de un modo contundente, a veces "agobiante", provocando, en no pocas ocasiones, recelos y desconfianzas en un mundo, como el universitario, endogámico y muy acostumbrado a "mirarse el ombligo" como procedimiento más profundo de "examen de conciencia". Esta última realidad toma más fuerza en los sectores más conservadores, sin que las Instituciones hayan sido capaces de provocar satisfactoriamente la conversión radical para garantizar el cambio necesario.

En septiembre de 1992, el entonces Consejo de Universidades adopta la resolución de desarrollar, durante el curso 1993-1994, el Programa Experimental de Evaluación de la Calidad del Sistema Universitario, con el principal objetivo de someter a prueba una metodología de evaluación institucional inspirada en las experiencias internacionales existentes.

Los objetivos previstos se consideraron cumplidos, especialmente los referidos a la construcción de un sistema de indicadores y a la evaluación de determinadas titulaciones, complementadas con elementos de juicio de expertos externos, aportados principalmente en relación con las actividades docentes y en menor intensidad respecto al campo de la investigación y la gestión (Comité Técnico de la Secretaría General del Consejo de Universidades, 1994). 
En el curso 1994-1995, la Comisión Europea desarrolla el Proyecto Piloto Europeo para la evaluación de la Calidad de la Enseñanza Superior, en el que el sistema español, junto a otros dieciséis países, participa evaluando dos titulaciones. Potenciar la necesidad de evaluación de la calidad en la enseñanza superior, introducir una dimensión europea en la evaluación de la calidad, enriquecer los procedimientos existentes y, contribuir a mejorar el reconocimiento de los diplomas y periodos de estudio entre los distintos países, fueron los objetivos de aquel proyecto. Este último objetivo fue, tal vez, la primera piedra para la construcción de lo que hoy conocemos como Espacio Europeo de Educación Superior.

Con tales experiencias previas, en diciembre de 1995, el entonces Ministerio de Educación y Ciencia establece, mediante el Real Decreto 1947/1995, el Plan Nacional de Evaluación de la Calidad de las Universidades (PNECU), con los objetivos de promover la evaluación de la calidad, elaborar metodologías homogéneas integradas en la práctica vigente de la Unión Europea y proporcionar información objetiva que pudiera servir de base para la adopción de decisiones de las distintas instituciones en el ámbito de su competencia. El Plan prevé una duración de cinco años en su desarrollo y establece una metodología mixta de autoevaluación y evaluación externa (Comité Técnico de la Secretaría General del Consejo de Universidades, 1994).

En la llamada evaluación externa, la participación de agentes ajenos al sistema universitario fue testimonial. Tal condición se consideraba satisfecha con la no pertenencia de los evaluadores, habitualmente académicos, a Universidades de la misma Comunidad Autónoma donde radicara la titulación evaluada, perdiéndose así una magnífica oportunidad de haber realizado el proceso con mayor credibilidad social, mediante la incorporación sistemática de evaluadores no pertenecientes al sistema universitario como miembros de los llamados Comités de Evaluación Externa. Además, aquéllos Ilegaron a asumir un papel de cómplices para ayudar a la mejora, antes que de auditores en la revisión del autoinforme, y no se posibilitaba la evaluación de resultados respecto a estándares de referencia.

En el Informe Global 1996-2000 del mencionado Plan, en cuyo desarrollo participaron la mayoría de las Universidades existentes (80\%-88\%, según anualidades), se recogen unas Reflexiones Finales en las que, junto a los logros habidos tales como el haber generado una cierta sensibilización hacia la calidad y su evaluación traducida en la creación de infraestructuras en las propias Universidades dedicadas a tal fin, amén del impulso en la creación de Agencias Autonómicas, se reconoce la escasa aceptación de la cultura de calidad por parte del personal universitario, previendo ya la dilatada duración del proceso de cambio (Consejo de Coordinación Universitaria, 2003).

En abril de 2001, mediante el Real Decreto 408/2001, de 20 de abril, se establece el II Plan de la Calidad de las Universidades, con una duración prevista de seis años para, siguiendo la línea iniciada en el primer Plan, fomentar la implantación de Sistemas de Calidad en las Universidades españolas; promover la participación de las Comunidades Autónomas propiciando la creación de Agencias de Evaluación autonómicas; desarrollar metodologías homogéneas con las existentes en la UE que permitieran establecer estándares para la acreditación; implantar un sistema de información que pueda servir de base para la toma de decisiones basado en la evaluación de resultados, y establecer un sistema de acreditación de programas formativos para garantizar la calidad en conformidad con estándares internacionales.

La aprobación en diciembre de 2001 de la Ley Orgánica, 6/2011, de 21 de diciembre, de Universidades (LOU) genera un nuevo orden de organización y coordinación de funciones en materia de calidad del sistema universitario que requiere, en aras de la transparencia y la 
seguridad jurídica, la derogación expresa del RD 408/2001 por el que se establece el II Plan de la Calidad de las Universidades, así como de sus disposiciones de desarrollo, para que los objetivos de dicho Plan sean impulsados desde la Agencia Nacional de Evaluación de la Calidad y Acreditación (ANECA) cuya creación preveía la mencionada Ley.

Como consecuencia de estas experiencias, las Universidades incorporaron a sus cargos unipersonales figuras encargadas de la gestión de la calidad, a la vez que se crearon unidades administrativas vinculadas, comúnmente conocidas como Unidades Técnicas de Calidad.

Sin embargo, el escepticismo, cuando no clara resistencia, de los miembros de la comunidad universitaria, especialmente del PDI, y la escasa permeabilidad del sistema no posibilitaron la impregnación de la cultura de la calidad en todos sus estratos o niveles y en todas las unidades.

Además, los Órganos de Gobierno de las Universidades (Equipos Rectorales, Consejos de Gobiernos y/o Claustros) no pocas veces "quedaban enterados" de los resultados de las actividades de evaluación, sin una clara asunción de compromisos sólidos respecto a las propuestas de mejora que se generaban en tales procesos, provocando el desánimo y la desilusión en los "miembros de a pie" de la comunidad universitaria implicados en aquéllas.

Por otro lado, las evaluaciones desarrolladas, con sus "metainformes" correspondientes, que globalizaban los resultados de las diversas titulaciones evaluadas, en general, carecieron de difusión y repercusión en la planificación estratégica de las instituciones.

Los Planes referidos permitieron detectar una serie de aspectos que necesitaban de un cambio urgente (absentismo, ausencia de indicadores, duración de estudios,... ); todos ellos "caldo de cultivo" para la mejora. Sin embargo, la Universidad, en la responsabilidad de su autonomía, no parece haber profundizado con la coherencia y contundencia que le posibilita su capacidad investigadora en la búsqueda de soluciones a dichos problemas.

\section{El proceso de Convergencia al EEES}

\section{El escenario europeo}

Paralelamente, en Europa, los procesos de convergencia avanzan en todos los ámbitos y la educación universitaria no queda fuera de ese movimiento. La Convención sobre el Reconocimiento de Cualificaciones de Educación Superior en Europa, celebrada en Lisboa (Conseil de I'Europe, 1997) y la posterior declaración de la Sorbona, celebrada en París en 1998 son la antesala de la Declaración de Bolonia, en junio de 1999, que fue el impulso decisivo para la construcción del Espacio Europeo de Educación Superior (EEES), abarcando en la actualidad a un total de 45 países.

EI EEES tiene como objetivo prioritario aumentar la compatibilidad y comparabilidad de los Sistemas de Educación Superior. La calidad y, más concretamente, "Ios Sistemas para la Garantía de la Calidad" pasan a ser el telón de fondo tanto de la Convención de Instituciones Europeas de Educación Superior (que tuvo lugar en el 2001 en la ciudad de Salamanca), como de la Declaración de Praga celebrada en el mismo año. Por otro lado, tras la Declaración de los Estudiantes de Göteborg (2001) se considera a los mismos como "socios competentes", cuya participación es indispensable para el éxito del proceso de convergencia. Es durante la Reunión de Instituciones de Educación Superior celebrada en Graz (2003), donde, además de ponerse de manifiesto la necesidad de incentivar la calidad institucional y rendir cuentas a la sociedad, se considera a los alumnos y al resto de grupos de interés agentes activos en la renovación del contexto universitario. 
El desarrollo de procedimientos y guías como mecanismo de revisión no se hizo esperar, representando la Declaración de Berlín en 2003 el contexto elegido para encargar dicha labor a la European Association for Quality Assurance in Higher Education (ENQA). Como resultado, dicha Red europea elaboró un informe en el que se establecieron los Criterios y Directrices para la Garantía de la Calidad en el Espacio Europeo de Educación Superior (ANECA, 2005).

\section{El proceso en España}

Ya en España, con objeto de dar respuesta a los requerimientos y exigencias europeas, la ya referida LOU del año 2001:

"... diseña la moderna arquitectura normativa que reclama el sistema universitario español para mejorar su calidad docente, investigadora y de gestión; fomentar la movilidad de estudiantes y profesores...e integrarse competitivamente junto a los mejores centros de enseñanza superior en el nuevo espacio universitario europeo que se está comenzado a configurar" (LOU, 6/2001, Exposición de motivos, apartado l, $5^{\circ}$ párrafo).

Los acuerdos en política de Educación Superior en Europa, derivados e Bolonia causaron la modificación de la LOU por la Ley Orgánica, 4/2007, de 12 de abril, y la promulgación del Real Decreto 1393/2007, de 17 de noviembre, por el que se establece la ordenación de las enseñanzas universitarias, parcialmente modificado por el Real Decreto 861/2010, de 2 de julio.

La Ordenación de las Enseñanzas Universitarias Oficiales, emprendida con la mencionada Ley Orgánica 4/2007, recoge la necesidad de establecer criterios que faciliten la evaluación, certificación y acreditación; considerando la garantía de calidad como un fin esencial en el ámbito de la política universitaria. Así mismo, este aspecto se ve reforzado en el documento publicado por el Ministerio de Educación y Ciencia (2006) bajo la denominación "Directrices para la elaboración de Títulos Universitarios de Grado y Máster".

El establecimiento de un sistema de créditos ECTS (European Credit Transfer System) mediante Real Decreto 1125/2003, de 5 de septiembre, la observancia de las recomendaciones de la ETCS Users' Guide (Organismo Autónomo Programas Educativos Europeos, 2010), el establecimiento del Suplemento al Título (Real Decreto 1044/2003, de 1 de agosto), la definición del Marco Español de Cualificaciones de Educación Superior (Real Decreto 1027/2011, de 15 de julio) y la observancia de los Criterios y Directrices para la Garantía de la Calidad en el EEES representan un conjunto normativo básico de referencia en el desarrollo del EEES en España (ANECA, 2005).

\section{Los nuevos Títulos: procesos de verificación e implantación}

Este marco constituye, hasta hoy, el escenario para la verificación y la acreditación de los títulos oficiales que, inicialmente, se definía como la "homologación de planes de estudios y de títulos" en la LOU 6/2001 y, en la que ya se señala que "... transcurrido el periodo de implantación de un plan de estudios, las Universidades deberán someter a evaluación de la ANECA el desarrollo efectivo de las enseñanzas" (Art. 35.5. ).

Tal mandato es suprimido en la modificación de la LOU por la Ley Orgánica 4/2007, de 12 de abril, apareciendo el término "verificación" para definir el hecho de que el plan de estudios se ajusta a las directrices y condiciones establecidas por el Gobierno de la nación (Modificación 31. Nueva redacción del art. 35, apart.2). 
En el posterior Real Decreto 1393/2007, de 29 de octubre, se establecen las normas para la verificación del plan de estudios (modificadas parcialmente por el Real Decreto 861/2010, de 2 de julio) y para el establecimiento del carácter oficial del título y su inscripción en el Registro de Universidades, Centros y Títulos (RUCT), acto que tendrá como efecto la consideración inicial de "título acreditado" (Art. 26.2). Este Real Decreto supone una transformación estructural y organizativa de los Títulos. Así, las enseñanzas adoptan la forma de "Grado", "Máster" y "Doctorado".

De acuerdo con lo establecido en el citado Real Decreto, los Planes de Estudio conducentes a la obtención de Títulos Oficiales serán verificados por el Consejo de Universidades, siendo la Agencia Nacional de Evaluación de la Calidad y Acreditación (ANECA), así como las Agencias Autonómicas competentes, las encargadas de evaluarlos y elaborar el informe correspondiente.

En cuanto a los cambios organizativos subsecuentes a esta Ley, se observa una transformación en la metodología docente, incentivándose el proceso de aprendizaje del estudiante "a lo largo de la vida" y fomentándose la movilidad, tanto en el contexto europeo como en otras áreas geográficas. Igualmente, se apuesta por un sistema de reconocimiento de créditos a través del cual serán considerados en el expediente del alumno sea cual sea la Universidad en la que haya cursado sus enseñanzas. El establecimiento de un Sistema de Garantía de Calidad (SCC) aparece por primera vez en la legislación española como parte de los planes de estudio y, en consecuencia, son condición de obligado cumplimiento para el proceso de Verificación y Acreditación de los Títulos.

Para su integración en este proceso, las universidades han adaptado sus Títulos a la nueva estructura del sistema, implantando los créditos ETCS, desarrollando las guías del título y las guías docentes a la luz de las recomendaciones de la ETCS User' Guide, han promovido y desarrollado programas de formación para el PDI a fin de dotarle de herramientas metodológicas y de evaluación, y han adecuado, en la medida de lo posible, sus infraestructuras, potenciando la utilización de las TIC en la enseñanza, o promoviendo los proyectos de innovación.

Pero no todas estas iniciativas han supuesto una transformación en el ejercicio de la actividad docente. En la mayoría de los casos ha habido autocomplacencia por la realización de tales actuaciones sin que se haya hecho una medición objetiva de los logros alcanzados. Entre las debilidades evidenciadas podemos señalar:

- El diseño de los nuevos planes de estudios han sido mayoritariamente definidos sobre la estructura y definición de materias anteriores, con defensa a ultranza de las "cuotas" de las que disfrutaban las diferentes áreas de conocimiento.

- Tampoco se ha avanzado en la efectiva coordinación entre materias, prevaleciendo el sentido de propiedad de la asignatura frente al de participación integrada en un proyecto común.

- Aún tienen una fuerte presencia metodologías y enfoques de evaluación incompatibles con Bolonia, lo que puede contrastarse a través de la consulta de la Web de cualquier universidad en no pocas asignaturas de cualquier título, especialmente de grado.

- Con la revisión de las normativas de progreso y permanencia se establecen diferencias en cuanto al tiempo en que pueden cursar una titulación, aunque queda mucho camino por recorrer para facilitar la enseñanza al estudiante a tiempo parcial.

- La comparación, intra e interuniversitaria es inexistente sin que se haya desarrollado una cultura de benchmarking, persistiendo anómalas situaciones en la práctica docente, 
y en el resto de ámbitos ligados con lo académico como la gobernanza o la gestión universitaria, susceptibles de ser abordadas y corregidas.

Así, es preciso señalar que mientras se exige a los docentes que aprendan nuevas metodologías, se sumerjan de lleno en una cultura de evaluación, y en un proceso de aprendizaje-enseñanza muy distinto al que venían realizando, los máximos responsables académicos no han apostado de lleno por ello. Antes de vernos inmersos en la crisis económica actual, algunas universidades españolas asumieron que la adaptación al EEES sería a "coste cero", lo que se tradujo en la negativa a la contratación de nuevo profesorado o a la división de los grupos docentes acordes a la nueva metodología. Tampoco ha existido un cambio en la forma de gestionar dentro de las universidades.

En lo que respecta a los datos sobre verificación de Títulos, durante el periodo 2008 a 2012 (ANECA, 2013), se encuentran verificados un total de 2680 Grados universitarios, 3500 Títulos de Máster y 86 Doctorados en el periodo 1011-2012 de acuerdo al RD 99/2011. Todos ellos deberán ser objeto de evaluación para renovar su acreditación (MECD, 2011).

\section{El seguimiento y la renovación de la acreditación de los Títulos oficiales}

Respecto al seguimiento de los títulos inicialmente acreditados, el citado RD 861/2010 modifica lo dispuesto con anterioridad en el RD 1393/2007 y, para su ejecución, establece que "... una vez iniciada la implantación de las enseñanzas correspondientes... a ANECA u órganos de evaluación que la ley de las Comunidades Autónomas determinen, llevarán a cabo el seguimiento del cumplimiento del proyecto contenido en el plan de estudios verificado". A este efecto, los órganos de evaluación en colaboración con el Ministerio y las correspondientes Comunidades Autónomas asumieron un protocolo, diseñado inicialmente por la Red Española de Agencias de Calidad Universitaria (REACU, 2010) en el que se definen unos criterios mínimos e indicadores básicos comunes para el procedimiento de seguimiento de planes de estudios. Es el denominado "Protocolo para el Seguimiento y la Renovación de la Acreditación de los Títulos Universitarios Oficiales, adoptado por la Comisión Universitaria para la regulación del Seguimiento y la Acreditación (CURSA)" (UEC, 2011).

Asimismo, el referido RD 861/2010, establece las normas para la renovación de la acreditación de los Títulos, señalando que:

"Antes del transcurso de seis años desde la fecha de su verificación inicial... los títulos universitarios oficiales de Grado y Doctorado, deberán haber renovado su acreditación de acuerdo con el procedimiento y plazos..." y para lo que "... se establecerán los protocolos de evaluación necesarios... de acuerdo con estándares internacionales de calidad..." (Modificación 11. Nueva redacción de los apartados 2 y 3 del art. 24).

El protocolo de renovación de acreditación de títulos oficiales, hoy día, no está definido. Aunque el título del documento antes mencionado incluye el concepto "renovación de la acreditación", el contenido del mismo no contempla ninguna referencia a dicho acto. Tampoco se han establecido los plazos para solicitud de la renovación de la acreditación que han de fijar las Comunidades Autónomas para sus ámbitos competenciales.

\section{El seguimiento de los Títulos}

Respecto al seguimiento de los Títulos, en la actualidad, las Agencias de Evaluación Universitaria han redactado y publicado protocolos para la aplicación en las universidades de su marco de competencia que se sustentan en el anteriormente comentado Protocolo para el Seguimiento y la Renovación de la Acreditación de los Títulos Universitarios Oficiales 
de la CURSA, y aprobados por el Consejo de Universidades y la Conferencia General de Política Universitaria.

Los propósitos del Seguimiento de los Títulos se centran en los siguientes:

1. Asegurar la ejecución efectiva de las enseñanzas conforme a lo recogido en el Plan de Estudios implantado, tal y como consta en el RUCT, junto a las modificaciones, en su caso, aprobadas por el Consejo de Universidades y autorizadas, en su caso, por las Comunidades Autónomas.

2. Asegurar la disponibilidad pública de la información pertinente y relevante a los diferentes agentes de interés del sistema universitario.

3. Detectar posibles deficiencias en el desarrollo de las enseñanzas y analizar las acciones realizadas para su subsanación.

4. Aportar recomendaciones y/o sugerencias de mejora en el transcurso de la Implantación del Plan de Estudios.

5. Identificar buenas prácticas para su difusión en el marco del sistema universitario español.

Por otro lado, la CURSA (2010) incorpora al seguimiento la definición de una serie de indicadores mínimos de resultados académicos, tres de los cuales ya figuraban en los Reales Decretos 1393/2007 y 861/2010. Éstos son:

- Tasa de Rendimiento.

- Tasa de Abandono.

- Tasa de Eficiencia.

- Tasa de Graduación.

En este contexto, en el que no hay que perder de vista la importancia de la temporalidad de la implantación de las nuevas enseñanzas universitarias, determinar el objeto y alcance del seguimiento resulta imprescindible. Así, se establece que éste abarca el periodo comprendido entre el inicio de la implantación del Plan de Estudios, hasta el momento en el que deba ser sometido a evaluación para renovar su Acreditación.

\section{Limitaciones del proceso de seguimiento}

Los criterios para el seguimiento se sustentan, pues, en los Criterios y Directrices para la Garantía de Calidad en el Espacio Europeo de Educación Superior (ANECA, 2005) elaborados por la European Association for Quality Assurance in Higher Education (ENQA). Así, los protocolos de seguimiento incorporan la revisión por pares, interna y externa, para la garantía de la calidad. El Informe de la ENQA, aun reconociendo la existencia de diferencias fundamentales entre las diversas Agencias acerca de la adecuada relación que deberían establecer las instituciones de educación superior y sus evaluadores externos, no dilucida la cuestión que queda abierta a la decisión de cada país participante.

Igualmente, el Informe ENQA prefiere el principio genérico al requisito específico y, como consecuencia, los criterios y directrices se centran más en lo que debería hacerse que en cómo debería lograrse.

El procedimiento para el seguimiento mantiene el modelo, ya establecido en las experiencias de evaluación de titulaciones previas al proceso de Bolonia, de que la garantía externa de la calidad tiene el principal propósito de la provisión de consejo y orientación en la búsqueda de mejoras frente a la concepción de aquélla como un asunto de "defensa del 
usuario" que requiere una clara distancia entre la agencia de garantía y las instituciones para revisar el cumplimiento efectivo del contrato social, entre la Universidad y los ciudadanos, que supone cada título verificado e inicialmente acreditado por su inscripción en el RUCT.

Sin lugar a dudas, consecuente con el principio de autonomía universitaria reconocido en la Constitución Española (art. 27.10), la responsabilidad primera respecto a la garantía de calidad en la educación superior descansa en la Universidad, y así se reconoce en el informe de la ENQA (ANECA, 2005), pero dicha autonomía exige un muy alto sentido de responsabilidad social, en correspondencia a la confianza concedida a la Universidad para el desempeño de sus funciones con eficacia y eficiencia al financiarse, fundamentalmente, con fondos públicos.

Entendemos que sería deseable una evolución en dicho modelo, al menos hacia posiciones intermedias, buscando un equilibrio entre la responsabilidad social exigible a las universidades, y la orientación para la mejora que faciliten las agencias. Ambas, responsabilidad y mejora, no sólo no son incompatibles sino que se complementan, e incluso pueden llegar a constituir una única meta a alcanzar por las universidades.

Sin duda, el diseño y futuro desarrollo del protocolo para renovación de la acreditación ofrece la posibilidad de ello y sería un error dejar pasar esta oportunidad para conseguir un adecuado ejercicio de control del cumplimiento de los logros de las Universidades en el desarrollo de sus programas inicialmente acreditados.

Respecto al desarrollo temporal del procedimiento de seguimiento, las universidades, atendiendo al protocolo aportado por la Agencia responsable del seguimiento, deben realizar informes anuales de seguimiento para cada uno de sus títulos que constarán de referencias de la información pública disponible y sus direcciones web, información de indicadores de resultados para constatación de logros intermedios, evidencias y logros de la implantación del sistema interno de garantía de la calidad y tratamiento de las recomendaciones recibidas de los agentes externos, evidenciando la existencia de mecanismos formales para el control y revisión, y en su caso modificación de sus planes de estudio.

Los informes realizados por los títulos sobre el desarrollo de su implantación servirán de base para el control del proceso de seguimiento, a realizar por las agencias u órganos de calidad externos, que, a su vez, generarán informes de carácter periódico, coexistiendo en el sistema español distintas frecuencias para su emisión: anuales, bienales, sin determinar... y serán de carácter individual para cada título y de carácter global comprendiendo los títulos y universidades pertenecientes a su ámbito de actuación.

En todos los casos, y en fidelidad a los criterios de transparencia y accesibilidad esenciales en el Proceso de Bolonia, los referidos informes de seguimiento tendrán carácter público.

La implantación del procedimiento de seguimiento ha dado lugar a un distinto estado de la cuestión según la agilidad de los agentes de garantía de calidad externos. Así frente a Comunidades Autónomas con Informes globales de seguimiento de sus universidades realizados en 2010/2012 y ya publicados, en otros casos no han sido emitidos, ni siquiera, los informes anuales de cada Título por parte de las Universidades.

Igualmente dispar es la visibilidad de la memoria del título y sus modificaciones, si las hubiere, lo que tiene una importancia clave ya que constituyen el documento de referencia del contrato social entre la universidad y la ciudadanía.

Por otro lado, entendemos que es manifiestamente mejorable la información requerida sobre indicadores de resultados para constatación de logros intermedios. Aun 
reconociendo que no se limita a las universidades en la consideración de los indicadores que estimen oportunos para su análisis y seguimiento, los propuestos inicialmente en los protocolos de seguimiento no garantizan el logro de los objetivos.

Ciertamente, algunos de ellos, como los de eficiencia del título, graduación e inserción laboral, sólo ofrecerán datos, como mucho, en el sexto año después de la verificación y puede ser que después de iniciarse el proceso de renovación de la acreditación. Además en ese momento, el valor de la tasa de eficiencia del título no será representativo de la realidad (dando valores más altos), pues es un indicador que demanda de más extensión temporal de análisis (un ciclo formativo, 4 años para el grado, más tres), para ajustarse a la realidad. Sí daría información válida el indicador de tasa de eficiencia de curso del título y año académico, especialmente los valores logrados en los tres últimos años de la implantación.

Igualmente sería de gran utilidad, por su segura repercusión en las tasas de graduación, especialmente, y en la de eficiencia en menor medida, el análisis del indicador de eficiencia por año académico y asignatura que permite detectar nítidamente los "cuellos de botella" en el progreso de los estudiantes.

El resto, rendimiento y abandono, así como otros considerados por las Universidades, tales como oferta/demanda, PDI doctor, créditos matriculados, etc., son, en unos casos, indicadores de proceso y posibles inductores de los futuros resultados, pero no garantes del éxito y, en otros, indicadores de contexto sin ninguna relación con los futuros logros.

\section{La renovación de la acreditación de los Títulos}

En las décadas de los ochenta y noventa se garantizaban a priori las titulaciones a través de la autorización y homologación de los títulos, mientras que, a partir del inicio del siglo, es a través de la evaluación de los proyectos (verificación) y la rendición de cuentas (acreditación).

Respecto a la acreditación de los títulos se ha producido un radical cambio de enfoque en el objetivo del proceso de acreditación.

Inicialmente, en 1999, como contribución al trabajo de seguimiento a la Declaración de la Sorbona, la Confederación de Conferencias de Rectores de la Unión Europea y la Asociación de Universidades Europeas (CRE) promueve el Informe Trends in Learning Structures in Higher Education (Tendencias en las estructuras de aprendizaje en Educación Superior) en el que se refleja que la evaluación de la calidad debe centrarse más en los "outputs" (aprendizaje, conocimiento y aptitudes requeridas) que en los "inputs" (enseñanza, programas de estudios) del sistema formativo (Haug, \& Kirstein, 1999).

Al amparo del II Plan de la Calidad de las Universidades se realiza un Proyecto de Acreditación de titulaciones de Grado, liderado por la Universidades Politécnicas de Cataluña y Valencia. Este Proyecto definía el establecimiento de procedimientos, criterios e indicadores para la acreditación y, aún más, establecía umbrales para los indicadores de resultados académicos que deberían cumplir las Universidades o programas para ser acreditados.

Paralelamente, en el III Foro de Reflexión de Almagro (Collado Yurrita y Vázquez Morcillo, 2001) sobre el ya citado II Plan de Calidad de las Universidades, se insiste en que la acreditación supone un cambio de paradigma y requiere de la implantación de sistemas de formación cuyo centro sean los conocimientos y actitudes adquiridas por el alumno, es decir, los resultados.

En la memoria de actividades correspondiente a 2003, la ANECA expresa que "la acreditación de títulos constituye el eje principal de las actuaciones de la Agencia", y que 
aquélla "se centra preferentemente en comprobar que los resultados de la formación son los adecuados y aseguran que las competencias que los estudiantes adquieren son las demandadas por la sociedad, el mercado laboral y por las necesidades de desarrollo científico y cultural de la sociedad en su conjunto" (ANECA, 2004).

Durante el periodo 2003-2006, ANECA diseña, desarrolla y aplica en experiencias piloto un modelo de acreditación con criterios, indicadores y estándares de calidad con el establecimiento de mínimos a cumplir por las titulaciones (umbrales), estableciendo estándares para algunos indicadores cuantitativos (ANECA 2004, 2005, 2006, 2007). Posteriormente, ANECA no ofrece información en sus Memorias sobre el programa de acreditación.

Sin embargo, es a partir del desarrollo de la LOU 6/2001 y, sobre todo del de la LOMLOU 4/2007, mediante el RD 1393/2007 que se define la acreditación de un título señalando que “...se basará en la verificación del cumplimiento del proyecto presentado por la Universidad..." (Preámbulo de la disposición). Se pasa así de un concepto de acreditación enfocado a resultados con umbrales de referencia a otro que se centra en "justificar el ajuste de la situación de lo realizado con lo propuesto en el proyecto presentado, o justificar las causas del desajuste y las acciones realizadas en cada uno de los ámbitos" (Anexo I, párrafo 1).

Este nuevo enfoque entendemos que queda lejos de alguno de los objetivos de la garantía de calidad de "medición del rendimiento del servicio público de la educación superior universitaria y la rendición de cuentas a la sociedad... la comparación... y la mejora de la calidad docente... de las Universidades" (art. 1., apart. 1.a.) al que la acreditación ha de contribuir.

Los trabajos desarrollados por la REACU relativos a la Renovación de la Acreditación de los Títulos Oficiales de Grado y Máster, en julio de 2011, ratifican el nuevo enfoque, predecible con el antecedente sobre seguimiento en el que el Protocolo de la CURSA utilizó como fuente primigenia el documento elaborado por la REACU en marzo de 2010, y señala que un informe favorable para la renovación de la acreditación indica que en el desarrollo de un título se cumplen los tres grandes criterios siguientes del documento referido:

a. Desarrollo del Plan de Estudios

b. Recursos humanos y materiales

C. Transparencia y resultados

Los resultados cuantitativos de las enseñanzas constituyen un aspecto, pero no el principal, de los criterios de acreditación que se proponen. Además, es posible que un Título se ajuste a la memoria previamente presentada y verificada, se cuente con los Recursos Humanos y materiales suficientes, y que todo ello sea transparente; pero eso, en ningún caso, garantiza la calidad de la educación ofrecida en dicho Título.

Por otro lado, y respecto a tales resultados, en las Memorias verificadas de los Títulos se estimaron unos valores sin que, externamente, se estableciera ningún valor de referencia o umbral para los mismos, cabiendo preguntarse si la estimación realizada por las Universidades, en muchos casos poco exigentes, y aceptada en el proceso de verificación ha de entenderse como satisfactoria, justificativa y suficiente para la inversión de recursos públicos destinados a ella.

La preparación para el ejercicio de actividades profesionales que exijan la aplicación de conocimientos y métodos científicos y para la creación artística, es una de las funciones de las Universidades que de esa u otra manera puede leerse en los primeros artículos del Estatuto 
de cualquier Universidad. Esto no es casual. Reproduce lo expresado en la LOU 6/2001, de 21 de diciembre, no modificado en la LOMLOU 4/2007, de 12 de abril de 2007, y reiterado en la misma Ley al señalar que "Las enseñanzas para el ejercicio de profesiones que requieren conocimientos científicos, técnicos o artísticos y la transmisión de la cultura son misiones esenciales de la Universidad" (art. 1, apart. 2.b.).

Por tanto, desarrollar los planes de estudios conforme a las memorias verificadas, de forma coherente y sin incidencias graves, con los recursos humanos, materiales y servicios necesarios para el desarrollo de las actividades previstas y poniendo en marcha los mecanismos necesarios para garantizar la disponibilidad de información pública objetiva y suficiente, el cálculo y valoración de la evolución de un núcleo de indicadores mínimo, el desarrollo del sistema interno de garantía de calidad, el análisis de las recomendaciones de los diferentes informes externos y la incorporación efectiva de las propuestas de mejora derivadas del seguimiento son responsabilidades inherentes al mandato legal definido entre sus misiones, y todas ellas deben ser cumplidas, no como un plus acreditativo de la calidad del Título sino como unas obligaciones ineludibles para asegurar la adquisición de las competencias por parte de los estudiantes.

\section{Discusión y conclusiones}

La renovación de la acreditación no debe sustentarse en el funcionamiento eficaz de las universidades, algo que, sin duda, las posibilitan para conseguir sus objetivos (indicadores de proceso), sino en la medición del rendimiento del servicio público de la educación superior universitaria y la rendición de cuentas a la sociedad, es decir, en la cuantificación de los resultados obtenidos en la misión esencial establecida por Ley: la formación de profesionales cualificados (indicadores de resultados) con transparencia y comparación entre instituciones.

Tememos que la acreditación, tal como parece que se está enfocando, tanto a través del proceso previo de seguimiento como por las propuestas que se conocen, derive en la legalización de los resultados previstos en las Memorias de verificación. En muchos casos son poco exigentes para la mejora de la institución y, en otros, además, escasamente ambiciosos y muy bajos en cuanto al éxito a alcanzar. Por otro lado, parece ser que algunas Agencias comienzan a manifestar que la acreditación de los Títulos va a consistir en una evaluación continua; esto es, cumplir los requisitos del seguimiento, año a año, introduciendo las mejoras por ellas propuestas.

Por todo ello, consideramos que cabría reflexionar sobre la conveniencia de la inversión pública a realizar en el caso de tan exiguos rendimientos o, tal vez, sobre la conveniencia de la agregación de instituciones o de titulaciones en búsqueda de una mayor rentabilidad y responsabilidad social.

Igualmente, consideramos que han de articularse mecanismos que permitan abordar, y superar, las graves deficiencias del sistema universitario detectadas en las experiencias de evaluación realizadas en el periodo 1995-2002 y que, en muchos casos, siguen presentes con la suficiente vitalidad para lastrar seriamente la eficacia y la eficiencia de la función docente encomendada por ley a las Universidades.

Además, tales deficiencias, desvían al sistema universitario del camino hacia algunos de los objetivos de Estrategia Universitaria 2015 (MECD, 2010) y representan un obstáculo para la correcta percepción de la responsabilidad social de las Universidades en el ámbito de la docencia. 


\section{Referencias}

Agencia Nacional de Evaluación de la Calidad y Acreditación, ANECA (2003). Memoria de actividades. Enero-diciembre 2003 [Informe]. Recuperado del sitio de internet de la Agencia Nacional de Evaluación de la Calidad y Acreditación, ANECA: http://www.aneca.es/Documentos-y-publicaciones/Memoria-de-actividades

Agencia Nacional de Evaluación de la Calidad y Acreditación, ANECA (2004). Un año de actividades de la ANECA. Enero-diciembre 2003 [Informe de actividades]. Recuperado del sitio de internet de la Agencia Nacional de Evaluación de la Calidad y Acreditación, ANECA: http://www.aneca.es/Documentos-y-publicaciones/Memoriade-actividades

Agencia Nacional de Evaluación de la Calidad y Acreditación, ANECA (2005). Criterios y directrices para la garantía de la calidad en el EEES [Informe]. (Traducido de Standards and guidelines for Quiality Assurance in the European Higher Education). Recuperado del sitio de internet de la Agencia Nacional de Evaluación de la Calidad y Acreditación, ANECA: http://www.aneca.es/Actividad-internacional/Documentosinternacionales-de-referencia/Criterios-y-estandares

Agencia Nacional de Evaluación de la Calidad y Acreditación, ANECA (2006). Memoria de actividades. Enero-diciembre 2005 [Informe de actividades]. Recuperado del sitio de internet de la Agencia Nacional de Evaluación de la Calidad y Acreditación, ANECA: http://www.aneca.es/Documentos-y-publicaciones/Memoria-de-actividades

Agencia Nacional de Evaluación de la Calidad y Acreditación, ANECA (2007). Un año de actividades de la ANECA. Enero-diciembre 2006 [Informe de actividades]. Recuperado del sitio de internet de la Agencia Nacional de Evaluación de la Calidad y Acreditación, ANECA: http://www.aneca.es/Documentos-y-publicaciones/Memoriade-actividades

Agencia Nacional de Evaluación de la Calidad y Acreditación, ANECA (2013). Informe sobre el estado de la evaluación externa de la calidad en las universidades españolas 2012 [Informe]. Recuperado del sitio de internet de Agencia Nacional de Evaluación de la Calidad y Acreditación, ANECA: http://www.aneca.es/Sala-deprensa/Noticias/2013/Estado-de-la-evaluacion-de-la-calidad-de-las-universidadesespanolas

Collado Yurrita, M.A. y Vázquez Morcillo, A. (2001). Conclusiones. En M.A. Collado Yurrita y A. Vázquez Morcillo (Coords.), El segundo plan de calidad de las universidades. III foro de reflexión, Almagro, mayo 2001 (pp. 427-430). Ciudad Real: Universidad de CastillaLa Macha.

Comité Técnico de la Secretaría General del Consejo de Universidades (1994). Programa Experimental de Evaluación del Sistema Universitario (Vol. I y II). Madrid: Consejo de Universidades.

Comunicado de Berlín (2003). Berlín 2003. "Educación Superior Europea". Comunicado de la conferencia de ministros responsables de la Educación Superior, mantenida en Berlín el 19 de semptiempre de 2003. Recuperado del sitio de internet de la Agencia Nacional de Evaluación de la Calidad y Acreditación, ANECA: http://www.aneca.es/Actividadinternacional/Documentos-internacionales-de-referencia/Comunicados-de-losministros-europeos 
Conseil de l'Europe (1997). Convention sur la reconnaissance des qualifications relatives à l'enseigmenent supérieur dans la región européenne (núm. 165). Lisbonne: Série de traités européens.

Consejo de Coordinación Universitaria (2003). Plan Nacional de Evaluación de la Calidad de las Universidades (PNECU). Informe global 1996-2000. Madrid: Ministerio de Educación, Cultura y Deporte (MECD).

Constitución Española, de 31 de octubre de 1978, aprobada por las cortes en sesiones plenarias del congreso de los diputados y del senado. (BOE núm. 311.1., de 6 de diciembre)

Declaración de Bolonia (1999). El espacio europeo de la enseñanza superior. Delaración conjunta de los ministros europeos de educación reunidos en Bolonia el 19 de junio de 1999. Recuperado del sitio de internet del Espacio Europeo de Educación Superior, EEES: http://www.eees.es/es/documentacion.

Declaración de la Sorbona (1998). Declaración conjunta para la armonización del diseño del Sistema de Educación Superior Europeo (a cargo de los cuatro ministros representantes de Francia, Alemania, Italia y el Reino Unido). Recuperado del sitio de internet del Espacio Europeo de Educación Superior, EEES: http://www.eees.es/es/documentacion.

Haug, G. \& Kirstein, J. (1999). Part I: Trends and issues in learning strutures in higuer education reports series. En G. Haug (Ed.), Trends in learning strutures in higher education. Recuperado de http://www.eua.be/eua/jsp/en/upload/OFFDOC_BP_trend_l.1068715136182.pdf

Ley Orgánica 6/2001, de 21 de diciembre, de Universidades. (BOE núm. 307, de 22 de diciembre).

Ley Orgánica 4/2007, de 12 de abril, por la que se modifica la Ley Orgánica 6/2001, de 21 de diciembre, de Universidades. (BOE núm. 89, de 13 de diciembre).

Ministerio de Educación, Cultura y Deporte, MECD (2001). II Plan de la Calidad de las Universidades 2001-2006. Recuperado del sitio de internet de Secretaría General Técnica, Subdirección General de Documentación y Publicaciones del Ministerio de Educación, Cultura y Deporte: http://www.ub.edu/iap/Legislacio/Pladequalitat.pdf

Ministerio de Educación, Cultura y Deporte, MECD (2006). Propuesta. Directrices para la elaboración de títulos universitarios de grado y master. Recuperado del sitio de internet de Secretaría General Técnica, Subdirección General de Documentación y Publicaciones del Ministerio de Educación, Cultura y Deporte http://firgoa.usc.es/drupal/files/directrices.pdf

Ministerio de Educación, Cultura y Deporte, MECD (2010). Estrategia Universitaria 2015. Recuperado del sitio de internet del Ministerio de Educación, Cultura y Deporte: http://www.mecd.gob.es/dctm/eu2015/2011-estrategia-2015espanol.pdf?documentld=0901e72b80910099

Ministerio de Educación, Cultura y Deporte, MECD (2011). Datos y cifras del Sistema Universitario Español. Curso 2011/2012. Recuperado del sitio de internet del Ministerio de Educación, Cultura y Deporte: http://www.mecd.gob.es/dms-static/6f7ca43de577-4db2-9999-823646043eff/2012-datos-y-cifras-11-12-pdf.pdf 
Organismo Autónomo Programas Educativos Europeos (2010). Guía de uso del ECTS (trad. Grupo de Expertos de Bolonia). (Reimpreso de ECTS user's guide, Comisión Europea, Ed., 2009, Bélgica: Bruselas).

Real Decreto 408/2001, de 20 de abril, por el que se establece el II Plan de la Calidad de las Universidades (Vigente hasta el 29 de noviembre de 2003). (BOE núm. 96 de 21 de abril).

Real Decreto 1044/2003, de 1 de agosto, por el que se establece el procedimiento para la expedición por las universidades del Suplemento Europeo al Título. (BOE núm. 218, de 11 de septiembre).

Real Decreto 1125/2003, de 5 de septiembre, por el que se establece el sistema europeo de créditos y el sistema de calificaciones en las titulaciones universitarias de carácter oficial y validez en todo el territorio nacional. (BOE núm. 224, de 18 de septiembre).

Real Decreto 1391/2003, de 17 de noviembre, por el que se deroga el Real Decreto 408/2001, de 20 de abril, por el que se establece el II Plan de la Calidad de las Universidades. (BOE núm. 285, de 28 de noviembre).

Real Decreto 1393/2007, de 29 de octubre, por el que se establece la ordenación de las enseñanzas universitarias oficiales. (BOE núm. 260, de 30 de octubre).

Real Decreto 861/2010, de 2 de julio, por el que se modifica el Real Decreto 1393/2007, de 29 de octubre, por el que se establece la ordenación de las enseñanzas universitarias oficiales. (BOE núm. 161, de 2 de julio).

Real Decreto 1027/2011, de 15 de julio, por el que se establece el Marco Español de Cualificaciones para la Educación Superior. (BOE núm. 185, de 3 de agosto).

Red Española de Agencias de la Calidad Universitaria, REACU (2010). Seguimiento de títulos oficiales [Documento de trabajo]. Recuperado del sitio de internet de Red Española de Agencias de Calidad Universitaria, REACU: http://www.unex.es/conoce-lauex/estructura-academica/centros/epcc/sgic/comision-de-garantia-de-calidad-delcentro/copy_of_otros-documentos/documentacion_COM_SATUO_15_abril_10-1.pdf

Red Española de Agencias de la Calidad Universitaria, REACU (2011). Renovación de la acreditación de los títulos universitarios oficiales de grado y máster [Documento de trabajo]. Recuperado del sitio de internet de Red Española de Agencias de Calidad Universitaria, REACU: http://web.ua.es/es/vrpeq/documentos/seguimiento/documento-reacu.pdf

Unidad de Evaluación de la Calidad, UEC (2011). Protocolo para el seguimiento y la renovación de la acreditación de los títulos universitarios oficiales (CURSA) (aprobado en julio de 2010 por el Consejo de Universidades y la Conferencia General de Política Universitaria. Recuperado del sitio de internet de Unidad de Evaluación de la Calidad, Universidad de Salamanca: http://web.ua.es/es/vr-peq/documentos/seguimiento/documentoreacu.pdf 


\section{Autores}

Jesús Miguel Muñoz Cantero

Es Profesor Titular de Universidad Entre sus líneas de trabajo e investigación destaca la evaluación de centros educativos en los diferentes niveles educativos, sobre los que ha escrito numerosos artículos y dirigidos diversas tesis doctorales en España y Brasil. Evaluador de diferentes agencias de calidad universitaria en España es también evaluador acreditado del Modelo EFQM y del Modelo Iberoamericano. Es miembro de la Asociación Amigos de la calidad http://amigosdelacalidad.es/ a los que se debe la discusión del documento que se publica

Facultad de Ciencias de la Educación. Campus de Elviña s/n. Universidade da Coruña. Coruña (Spain) 15071. Teléfono: (+34) 981167000. Ext.1799 Fax. (+34) 981167153. e-mail: jesus.miguel.munoz@udc.es

\section{Carmen Pozo Muñoz}

Es Profesora Titular del Área de Psicología social. Sus líneas principales de investigación son la Evaluación de Programas, Intervención social y la calidad en el ámbito académico. Ha dirigido diversos proyectos de investigación sobre calidad docente y sistemas de garantía de calidad, que han derivado en numerosas publicaciones. Fue Directora de la Unidad de Calidad de la Universidad de Almería y en la actualidad es Presidenta de la Asociación de Amigos de la Calidad

Facultad de Humanidades y Psicología. Universidad de Almería. 04120 Almería. Teléfono: (+34)950015728. E-mail: cpozo@ual.es 